
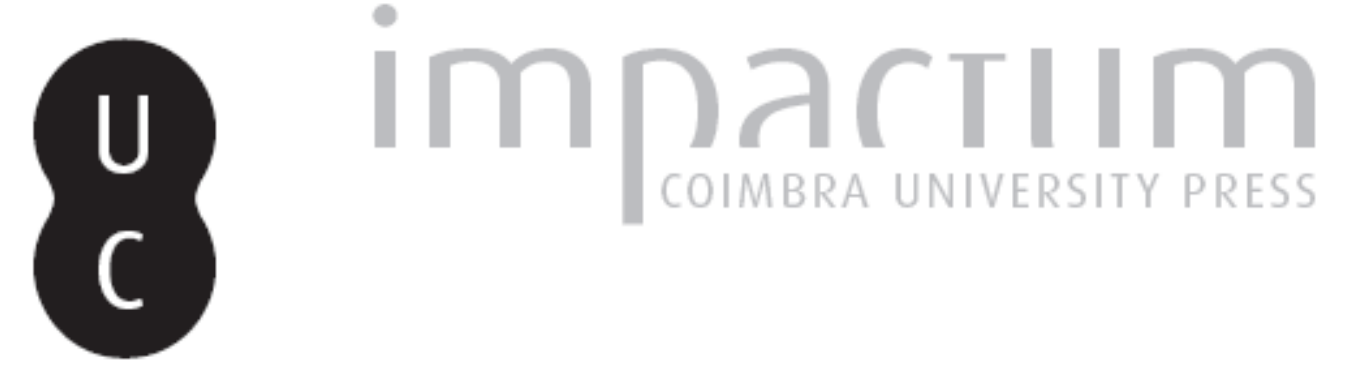

\title{
[Recensão a] Adamastor e dintorni. In ricordo di Antonio Tabucchi, con un frammento inedito, a cura di Valeria Tocco
}

Autor(es): Bettini, Clelia

Publicado por: Imprensa da Universidade de Coimbra

URL

persistente:

URI:http://hdl.handle.net/10316.2/42419

DOI:

DOI:https://doi.org/10.14195/0870-8584_9_13

Accessed : $\quad$ 26-Apr-2023 13:14:11

A navegação consulta e descarregamento dos títulos inseridos nas Bibliotecas Digitais UC Digitalis, UC Pombalina e UC Impactum, pressupõem a aceitação plena e sem reservas dos Termos e Condições de Uso destas Bibliotecas Digitais, disponíveis em https://digitalis.uc.pt/pt-pt/termos.

Conforme exposto nos referidos Termos e Condições de Uso, o descarregamento de títulos de acesso restrito requer uma licença válida de autorização devendo o utilizador aceder ao(s) documento(s) a partir de um endereço de IP da instituição detentora da supramencionada licença.

Ao utilizador é apenas permitido o descarregamento para uso pessoal, pelo que o emprego do(s) título(s) descarregado(s) para outro fim, designadamente comercial, carece de autorização do respetivo autor ou editor da obra.

Na medida em que todas as obras da UC Digitalis se encontram protegidas pelo Código do Direito de Autor e Direitos Conexos e demais legislação aplicável, toda a cópia, parcial ou total, deste documento, nos casos em que é legalmente admitida, deverá conter ou fazer-se acompanhar por este aviso.

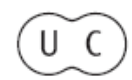




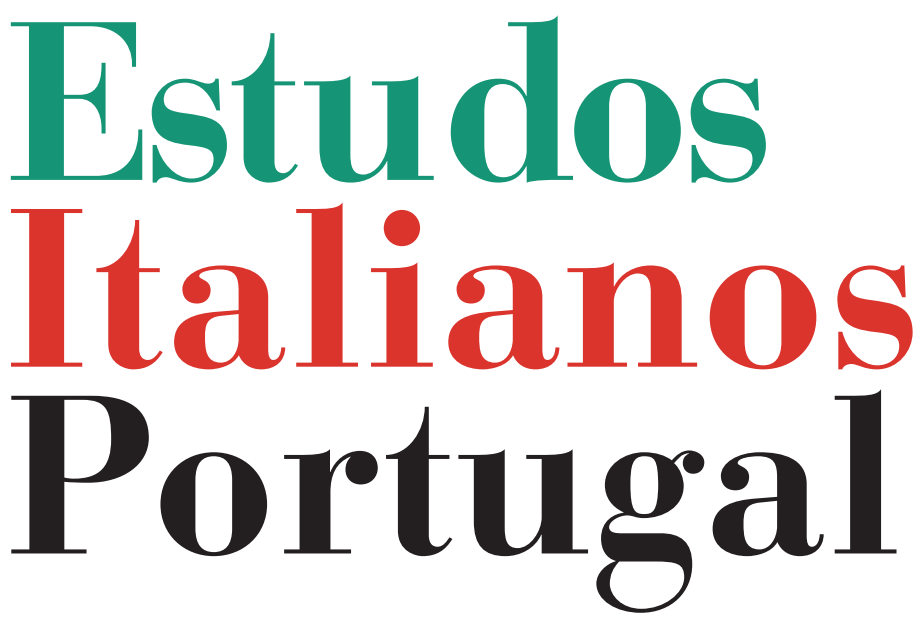

Instituto

Italiano

de Cultura

de Lisboa

Nova Série

$\mathbf{N}^{\circ} 9$ 
appunti: un piccolo museo di nostalgie. Li conserva tuttora gelosamente, questi foglietti, quasi fossero più importanti del testo stesso, e li assimila ai "paratesti", espressione che lì per lì fece sorridere Tabucchi: "Para che?", gli chiese, "fra l'ironico e il finto stupito" (p. 10) ${ }^{1}$.

Mi piace pensare che a un certo punto Tabucchi si sia fermato e gli abbia detto, col suo modo di fare schietto tipico dei toscani: "monta su Roberto, io ho fatto tanti chilometri, adesso, se non ti dispiace, tocca a te guidare".

A lui è affidata, infatti, la cura di questo volume.

RICCARDO GRECO

Adamastor e dintorni. In ricordo di Antonio Tabucchi, con un frammento inedito, a cura di Valeria Tocco, Pisa, ETS, 2013, pp. 185.

Come spesso piace fare ai filologi, vorrei partire da una riflessione su una parola per spiegare l'importanza di questo prezioso volume che Valeria Tocco ci ha regalato a un anno dalla morte di Antonio Tabucchi e che raccoglie gli interventi proferiti in occasione di due giornate dedicate alla memoria dello scrittore, tenutesi una all'Università di Pisa e l'altra all'Università di Genova. La parola in questione è, appunto, filologia. È un termine che indica una scienza imprescindibile per chi ama la letteratura, come la medicina o la fisica lo sono per chi ama la vita. È una scienza che spesso incute timore, soprattutto agli studenti che devono studiarla, e che richiede silenzio, tempo, contemplazione, tutte cose che al giorno d'oggi sono divenute merce rara e preziosa. L'etimologia di questa parola è invece molto semplice e dunque universale: essa indica l'amore (filía) per la parola (lógos). E nell'attività del filologo l'amore per la parola giunge spesso al parossismo, egli può arrivare a trascorrere giornate intere su un unico frammento di discorso, per decriptarne il senso nascosto, per ricostruirne un pezzetto mancante o per metterlo in relazione con l'universo che lo contiene. $\mathrm{E}$ in fin dei conti, la sua professione non differisce molto da quella dello scrittore che attraverso il logos, inteso poi nel suo senso più profondo di pensiero che nasce dal verbo $e$ in esso si esprime, crea la sua arte. Il sentimento di filía che lega filo-

${ }^{1}$ Ibidem, p. 10. 
logia e creazione letteraria ha dato origine a un sodalizio duraturo che ha permesso a entrambe di continuare ad esistere: senza i filologi - dall'antichità fino a oggi - non avremmo pressoché nulla di tutto quello che consideriamo letteratura e, senza la letteratura, è chiaro, i filologi farebbero, che so, magari i gelatai.

Ecco perché la scelta di dedicare la giornata pisana in ricordo di Tabucchi alla sua attività di filologo e studioso di letteratura si rivela fondamentale, perché fa luce su una parte essenziale della sua vita intellettuale che ha avuto "ricadute sia sulla carriera accademica, sulla sua attività scientifica e sulla sua produzione letteraria", come scrive, a ragione, Valeria Tocco nella sua introduzione al volume. Docente di Letteratura Portoghese all'Università di Pisa, Valeria Tocco ricorda come a Pisa tutto è cominciato: la laurea con Luciana Stegagno Picchio, il Perfezionamento in Normale e la nascita di un primo nucleo dinamico di giovani lusitanisti che avrebbero contribuito a dare lustro a una branca degli studi letterari sino ad allora considerata liminale, lontana e sconosciuta come il Portogallo claustrofobico degli ultimi anni del Salazarismo.
E lo studio tabucchiano del gigante Adamastor in chiave comparatistica che andava a fotografare l'anello che non tiene della retorica di regime, così come gli innovativi saggi sull'allora pressoché misconosciuto Fernando Pessoa sono solo alcuni dei frutti degli anni pisani del Tabucchi studioso e professore universitario, testi che hanno segnato - come ricorda ancora Tocco - intere generazioni di lusitanisti, compresa chi scrive. $\mathrm{E}$ in questo volume s'incrociano $\mathrm{i}$ ricordi di tanti studiosi che hanno conosciuto Antonio Tabucchi a Pisa e a Genova: colleghi, ex-allievi $\mathrm{o}$, più semplicemente, amici.

E il primo ricordo è quello di Valeria Bertolucci Pizzorusso, fine studiosa di quella poesia galegoportoghese che è la prima e arcaica espressione di tutto l'universo letterario lusitano. Bertolucci rievoca l'immagine di un Tabucchi giovane studente di Stegagno Picchio e amante della poesia e forse poeta anch'egli, la bellissima rivista "Quaderni portoghesi”, edita proprio a Pisa, i legami con Silvio Guarnieri e la necessità di un nuovo tipo di letteratura engagé. E proprio sull'impegno dell'intellettuale versa il vibrante intervento di Salvatore Settis che ricorda come Tabucchi 
abbia sempre saputo restare dentro la polis, criticandola dal suo interno e usando un linguaggio temerario ma diretto a tutti i cittadini, capace di portare avanti battaglie scomode e pericolose, in nome della giustizia e della cultura intesa come bene comune e non come orpello da disprezzare o, peggio ancora, come una serie di mostrine appuntate sulla divisa che s'indossa.

L'ispanista Francesco Guazzelli traccia un ritratto scanzonato e melancolico a un tempo degli anni pisani di Tabucchi, descrivendo un gruppo di giovani amici affascinati dalla filologia e dalla letteratura che grazie al loro dinamismo hanno fatto la fortuna di tanti studenti che, anni dopo, hanno frequentato le serie biblioteche e i tristi marchingegni per la lettura dei microfilm, arnesi obsoleti simboli di un tempo ormai, forse, perduto per sempre. E di amici parla anche il rumenista Bruno Mazzoni, ricordando le numerose affinità elettive tra Tabucchi e scrittori e intellettuali rumeni, fra i quali Mircea Eliade con la sua permanenza a Lisbona e il suo interesse per il Portogallo, il poeta Gregore Arbore Popescu che Tabucchi conobbe in Normale negli anni Settanta e, infine, lo scrittore esule Norman
Manea, a cui lo legava una sincera amicizia nata al prestigioso Bard College di New York. Amicizie che rivelano un profondo interesse per la Romania e la sua cultura, presente non di rado anche nella sua produzione letteraria, un paese che condivide con il Portogallo quella sorta di stigma periferico che, a ben vedere, non fa che aumentarne il fascino agli occhi di coloro che nutrono per la letteratura una curiosità e un interesse sinceri.

Alessandro Martinengo ci riporta invece a Pisa e al Siglo de oro spagnolo, ripercorrendo a distanza di molti decenni l'analisi di Tabucchi della figura del gigante camoniano, attraverso il dialogo con le sue fonti classiche e, soprattutto, Góngora, dando prova di come certe "piste" - quelle più audaci - continuino a suggerire sempre nuovi itinerari per coloro che siano curiosi di tornare a percorrerle. E di curiosità scrive anche Remo Ceserani, di quel particolare tipo di curiosità che appartiene solo ai grandi, a coloro che sono i più severi giudici di sé stessi. Ceserani ripercorre alcune fasi della vita e dell'opera di Tabucchi, mettendo in luce uno - a mio parere - dei suoi meriti maggiori: il coraggio di abbattere gli steccati tra generi 
diversi di letteratura e tra le diverse forme di comunicazione, spingendosi sempre oltre, laddove una sorta di necessitas lo obbligava ad andare, in barba alle aspettative o ai pregiudizi di cui inevitabilmente gli altri - critici, colleghi o semplici lettori - sono vittime. Blanca Periñan pone l'accento su un altro aspetto, per così dire, anticonformista di Antonio Tabucchi, ovvero la sua abilità nel giocare con le parole e nel mescidare lingue, dialetti, espressioni idiomatiche, innestandole alla perfezione in quella sua lingua tanto amata, che egli stesso definiva come "la nostra lingua che ho cercato di difendere scrivendola" (p. 84). E di un particolare tipo di scrittura, quella giornalistica, ci parla Thea Rimini, studiosa dell'opera di Tabucchi, che mette in luce in modo acuto e originale come i numerosi articoli pubblicati sul "Corriere della Sera" e su "Repubblica" siano rivelatori "della sua poetica e distillano la sua idea di letteratura: una letteratura interrogativa, aliena alle logiche di intrattenimento che si affermano sempre di più a partire dalla metà degli anni Ottanta" (p. 96.) E un altro giovane studioso, Giuseppe Marcocci, frequentatore abituale di tribunali della fede ed altre ame- nità lusitane dell'età moderna, si sofferma su una di quelle battaglie a favore dei più deboli che, da sempre, si sono intrecciate all'attività del Tabucchi filologo e scrittore: il sostegno alle popolazioni rom vittime di reiterate ingiustizie. E concordo a pieno con Marcocci, che sa bene quel che dice quando scrive: "in fondo, credo che Antonio fosse convinto che il mondo sarebbe migliore se fossimo tutti un po' più zingari e meno inquisitori” (p.102).

L'ultima parte del volume di cui scriviamo raccoglie alcuni interventi della giornata in memoria di Tabucchi che si è svolta all'Università di Genova, ateneo in cui lo scrittore insegnò dal 1978 al 1990, ultima tappa del suo percorso prima di approdare a Siena, dove terminerà la sua carriera di docente. Mentore di questa giornata è Roberto Francavilla che ha cominciato a studiare e ad amare il mondo lusofono da allievo di Tabucchi proprio a Genova, è divenuto poi suo collega a Siena e a Genova ha finito col tornare, sulla cattedra del suo professore, come egli stesso spiega nella seconda appendice del suo intenso e emotivo intervento. Francavilla raccoglie alcune riflessioni nate nel corso di questo 
primo anno di assenza del suo maestro, sui molti "demoni dei crocicchi" che, come nel celebre blues di Robert Johnson, aspettano chi voglia incontrarli, per scombinare ogni cosa e rendere la vita degna di essere davvero vissuta: Fernando Pessoa, la letteratura capoverdiana e la saudade.

Il primo intervento genovese è a carico dell'italianista Giorgio Bertone che rende omaggio al collega Tabucchi riflettendo su cinque punti a suo parere essenziali per comprendere perché "la scrittura di Tabucchi ci ha tanto sorpreso [...] e in seguito comunque affascinato" (p. 112). Luigi Surdich si sofferma invece sull'Inquietudine tabucchiana e sulla quête che ne deriva, un percorso di ricerca che i suoi personaggi intraprendono e che resta sempre senza risposte definitive perché essa “è il mezzo, è anche il fine" (p. 131). Del mistero, elemento connaturato all'Inquietudine, scrive Francesco De Nicola, proponendo un'analisi di Tabucchi come scrittore di romanzi d'indagine dove si riflette sulla legalità e sulle sue violazioni, istituendo un parallelo fra l'autore de Il filo dell'orizzonte e scrittori come Sciascia, Gadda e Pirandello. Silvia Zangrandi ricostruisce invece la presenza del modo fantastico nel- la narrativa di Antonio Tabucchi, analizzando quei testi dove il confine tra realtà e sogno è più labile e dove è possibile rintracciare una "meditazione sugli incubi e sulle tensioni dell'uomo moderno" (p. 145). Chiude la sezione genovese un testo di Eugenio Bonaccorsi che mette in luce un Tabucchi meno studiato e commentato, quello dei testi per il teatro.

Oltre al Tabucchi filologo, in questo volume-omaggio emergono molteplici aspetti di quello che è stato senza dubbio uno dei grandi scrittori del Novecento. Nel leggerlo sembra di guardare dentro a un caleidoscopio dove $\mathrm{i}$ colori cambiano in continuazione, ma si mantengono inalterati $\mathrm{i}$ contorni essenziali delle diverse figure che si susseguono davanti al nostro occhio vigile. In particolare, c'è una figura che ritorna spesso nei ricordi fissati su queste pagine, quella di sua moglie Maria José de Lancastre. È proprio grazie a lei che questo Adamastor e dintorni si arricchisce di un piccolo gioiello, un frammento inedito di Antonio Tabucchi dal titolo Quaderno greco e che ci dà l'illusione che questo grande scrittore continui a mandarci messaggi, da Sirio o dal Cane Maggiore. CLELIA BETTINI 